\title{
Rewriting the rules of inheritance
}

A new study published in Nature Biotechnology describes a novel approach that alters the rules of chromosome segregation in Caenorhabditis elegans resulting in non-Mendelian inheritance of entire genomes. Engineered non-Mendelian inheritance could facilitate a variety of applications from the study of gene regulation to synthetic biology.

With this potential in mind, the authors set out to disrupt the normal patterns of inheritance by manipulating the pulling strength of the mitotic spindle, an apparatus that separates and segregates sister chromatids evenly during mitotic cell division. To this end, they engineered a strain of C. elegans that overexpresses $G$ protein regulator 1 (GPR1), which controls the pulling force of the spindle, and used time-lapse microscopy to monitor the segregation of chromosomes during zygotic mitosis in transgenic and wild-type embryos. As expected, wild-type embryos formed a single bipolar spindle, and maternal and paternal chromosomes were segregated equally to produce identical daughter cells. By contrast, most GPR1-overexpressing embryos formed two monopolar spindles, each of which contained the chromosomes from one parent only, resulting in segregation of the maternal and paternal chromosomes into different daughter cells. Notably, this aberrant spindle formation was restricted to zygotic mitosis, as normal spindle formation was observed in subsequent cell divisions.
The daughter cells of C. elegans zygotes are known to give rise to separate cell lineages, with the anterior cell (AB lineage) developing into somatic cells and the posterior cell (P1 lineage) developing into the germ line and certain somatic cells. To monitor the segregation of the paternal and maternal chromosomes into these lineages, the researchers mated GPR1-overexpressing worms that also expressed a red fluorescent marker with worms expressing a green fluorescent marker. They observed two types of first-generation offspring (F1): worms that expressed both the green and the red markers in all tissues (resulting from equal segregation of maternal and paternal chromosomes during zygotic division) and 'hybrid' worms that expressed only the green or the red marker in different tissues, indicating that each cell of the body contained the DNA of only one parent, owing to unequal segregation of chromosomes during zygotic division. Most of the hybrid worms expressed the maternal marker in cells belonging to the $\mathrm{AB}$ lineage (such as neurons), and the paternal marker in cells belonging to the P1 lineage (such as muscle).

As the germ line is derived only from the P1 lineage, the authors reasoned that uneven segregation of chromosomes into different lineages in F1 worms should give rise to non-Mendelian inheritance patterns in the second generation (F2), which are produced by self-fertilization of the F1 generation. Indeed, as expected, F1 worms exhibiting lineage-specific expression of markers passed only the marker that isexpressed in the P1 lineage to F2 offspring. Thus, F2 offspring contained the DNA of only the paternal or maternal grandparent, illustrating a non-Mendelian pattern of inheritance.

Finally, by following the co-segregation of alleles that were situated on different chromosomes and had clearly distinguishable phenotypes, the authors confirmed that this inheritance pattern applied to the entire genome, not just to individual genes.

The authors suggest that this approach could enable novel studies, such as the generation of lineagespecific knockouts, and prove particularly useful for studies of epigenetic signalling and transgenerational inheritance.

\section{Denise Waldron}

\begin{abstract}
ORIGINAL ARTICLE Besseling, J. \& Bringmann, H. Engineered non-Mendelian inheritance of entire parental genomes in C. elegans. Nat. Biotechnol. http://dx.doi.org/10.1038/nbt.3643 (2016)
\end{abstract}

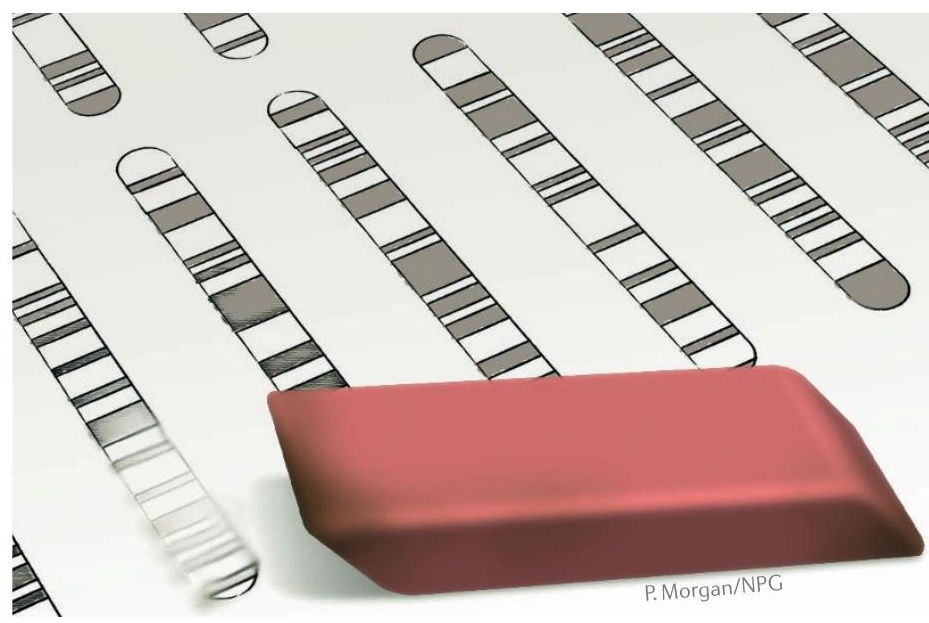

